\title{
INDECOMPOSABLE COALGEBRAS, SIMPLE COMODULES, AND POINTED HOPF ALGEBRAS
}

\author{
SUSAN MONTGOMERY
}

(Communicated by Lance W. Small)

\begin{abstract}
We prove that every coalgebra $C$ is a direct sum of coalgebras in such a way that the summands correspond to the connected components of the Ext quiver of the simple comodules of $C$. This result is used to prove that every pointed Hopf algebra is a crossed product of a group over the indecomposable component of the identity element.
\end{abstract}

\section{INTRODUCTION}

A basic structure theorem for cocommutative coalgebras asserts that any such coalgebra $C$ is a direct sum of its irreducible components; as a consequence, it can be shown that any pointed cocommutative Hopf algebra is a skew group ring of the group $G$ of group-like elements of $H$ over the irreducible component of the identity element. These results were proved independently by Cartier and Gabriel and by Kostant in the early 1960's; see [Di, S1]. This paper is concerned with versions of these results for arbitrary coalgebras and for arbitrary (pointed) Hopf algebras.

In fact much is already known about the coalgebra problem. In $1975 \mathrm{Ka}-$ plansky [K] showed that any coalgebra $C$ is (uniquely) a direct sum of indecomposable ones; moreover when $C$ is cocommutative, the indecomposable components are irreducible. In 1978 Shudo and Miyamoto [ShM] defined an equivalence relation on the set of simple subcoalgebras of $C$ and showed that the equivalence classes correspond to the indecomposable summands. A weaker version of this equivalence relation was studied recently in [XF].

In this paper we first refine what is known about the indecomposable components of $C$ by proving a dual Brauer-type theorem: each component corresponds to a connected component of $\Gamma_{C}$, the Ext quiver of the simple (right) comodules of $C$ (Theorem 2.1). In fact $\Gamma_{C}$ can be viewed as a directed graph whose vertices are the simple subcoalgebras of $C$; in this formulation it extends the equivalence relation of $[\mathrm{ShM}]$. We give a direct proof of these facts, and so give an alternate proof of the results of $[\mathrm{K}]$ and $[\mathrm{ShM}]$. We use only a few basic coalgebra properties (such as local finiteness) and a theorem of Brauer on finite-dimensional algebras.

Received by the editors September 21, 1993 and, in revised form, November 24, 1993; this paper was presented to an AMS Central Section Meeting at College Station, Texas, in October 1993.

1991 Mathematics Subject Classification. Primary 16W30, 16S35.

(C)1995 American Mathematical Society 
Secondly, we apply the coalgebra result and prove that for any pointed Hopf algebra $H$, there is a normal subgroup $N$ of the group $G$ of group-like elements such that $H$ is a crossed product of $G / N$ over the indecomposable component of the identity element of $H$ (Theorem 3.2).

When $H$ is pointed, $\Gamma_{H}$ can be described completely if all the skew-primitive elements are known; see Examples 1.3-1.5.

One motivation for our use of $\Gamma_{C}$ comes from a recent paper of Chin and Musson [CM], in which they study the duals of certain quantum groups. For a Noetherian Hopf algebra $H$, they wish to generalize the definition of the hyperalgebra $H^{\prime}$ of $H$. To do this, they begin with the maximal ideal $m_{0}=$ ker $\varepsilon$ of $H$ and consider the "clique" of cofinite maximal ideals $m$ which are equivalent to $m_{0}$ via a sequence of other such ideals which are "linked" to $m$ in the sense of non-commutative Noetheran ring theory (see [GW]). This clique of maximal ideals of $H$ dualizes to a set $\mathscr{S}_{0}$ of simple subcoalgebras of $C=$ $H^{0}$, with the links dualizing to a relationship among the simple subcoalgebras of $C$. The set $\mathscr{S}_{0}$ determines a subcoalgebra $D$ of $C$ which is the "new" hyperalgebra.

Here we begin with an arbitrary coalgebra $C$ and define the analog of links directly on the simple subcoalgebras of $C$; this gives us the graph $\Gamma_{C}$ as mentioned above. The set $\mathscr{S}_{0}$ of [CM] is then the connected component of $k \varepsilon$ in $\Gamma_{C}$.

It was also shown in [CM] that if $H=\mathscr{O}_{q}(S L(2))$, then $C=H^{0}$ is a crossed product over the "hyperalgebra" $D$. This crossed product result suggested to us that a similar result might be true more generally.

\section{Simple subcoalgebras and the Quiver $\Gamma_{C}$}

We first review a few definitions; we follow [S1] and [M2, Chapter 5]. Let $k$ be a field. $C$ will denote a $k$-coalgebra with comultiplication $\Delta: C \rightarrow C \otimes C$ and counit $\varepsilon: C \rightarrow k$. A basic fact is that $C$ is locally finite in the sense that any finite subset of elements of $C$ lies in a finite-dimensional subcoalgebra $D$ of $C . C$ is simple if it has no proper subcoalgebras; equivalently the linear dual $C^{*}$ is a finite-dimensional simple $k$-algebra. $C$ is irreducible if it has a unique simple subcoalgebra.

For any $C$, the group-like elements in $C$ are the set $G(C)=\{x \in C \mid x \neq 0$ and $\Delta x=x \otimes x\}$; necessarily $\varepsilon(x)=1$ for $x \in G(C)$. Note that a simple subcoalgebra $D$ of $C$ is one-dimensional $\Leftrightarrow D=k x$ for some $x \in G(C)$. A coalgebra is pointed if all of its simple subcoalgebras are one-dimensional.

For $x, y \in G(C)$, the $x, y$-primitive elements in $C$ are the set $P_{x, y}(C)=$ $\{c \in C \mid \Delta c=c \otimes x+y \otimes c\}$; necessarily $\varepsilon(c)=0$ for $c \in P_{x, y}(C)$. Note that $k(x-y) \in P_{x, y}(C)$; an $x, y$-primitive element $c$ is non-trivial if $c \notin k(x-y)$. If $x=y=1$, the 1,1-primitives are simply called primitive; otherwise they are called skew primitive.

We first define the quiver using the simple subcoalgebras of $C$. Recall from [S, 9.0] that the wedge of two subspaces $D$ and $E$ of $C$ is defined to be

$$
D \wedge E=\Delta^{-1}(C \otimes E+D \otimes C) .
$$

Note that if $D$ and $E$ are subcoalgebras, then it is always true that $D \wedge E \supseteq$ $D+E$. 
1.1 Definition. Let $\mathscr{S}$ be the set of simple subcoalgebras of a coalgebra $C$.

(1) The quiver $\Gamma_{C}$ is given as follows:

(V) the vertices of $\Gamma_{C}$ are the elements of $\mathscr{S}$;

(E) there exists an edge $S_{1} \rightarrow S_{2}$ for $S_{i} \in \mathscr{S}$, $\Leftrightarrow S_{2} \wedge S_{1} \neq S_{1}+S_{2}$.

(2) $C$ is called link-indecomposable (L.I.) if $\Gamma_{C}$ is connected (as an undirected graph).

We will also say that $S_{1}$ and $S_{2}$ are linked if $S_{1} \rightarrow S_{2}$ or $S_{2} \rightarrow S_{1}$, and that $S_{1}$ and $S_{2}$ are connected (or $S_{1} \sim S_{2}$ ) if they are in the same connected component of $\Gamma_{C}$.

1.2 Remark. When $C$ is pointed and $x, y \in G(C)$, we will write $x \rightarrow y$ if $S_{1} \rightarrow S_{2}$, where $S_{1}=k x$ and $S_{2}=k y$. It is known in this case that $x \rightarrow y \Leftrightarrow$ there exists a non-trivial $x, y$-primitive element. This follows by the TaftWilson theorem [M2, 5.4.1]; it is also not difficult to show directly. If $C$ is a bialgebra and $x \rightarrow y$, then also $x z \rightarrow y z$ and $z x \rightarrow z y$, for all $z \in G(C)$. For, if $\Delta c=c \otimes x+y \otimes c$, then $\Delta(c z)=\Delta c \Delta z=c z \otimes x z+y z \otimes c z$, and so $x z \rightarrow x z$. Similarly $z x \rightarrow z y$ using $z c$.

We note that the connection between skew-primitive elements and links was observed in [CM].

1.3 Example. Let $C=U(g)$, the enveloping algebra of a non-zero Lie algebra $g$. Then $\mathscr{S}=\{k 1\}$. Since $P_{1,1}(C)=g \neq 0, \Gamma_{c}$ is given by a loop:<smiles>I[V]C1CCCCCC1</smiles>

1.4 Example. Assume that $k$ contains a primitive $n$th root of unity $\lambda$ and let $C=H_{n^{2}}$, the Taft algebra of dimension $n^{2}$ [Tf]. This is a non-commutative, non-cocommutative Hopf algebra of dimension $n^{2}$ (the case $n=2$ was defined by Sweedler). As an algebra,

$$
H_{n^{2}}=k\left\langle g, x \mid g^{n}=1, x^{n}=0, x g=\lambda g x\right\rangle
$$

with coalgebra structure given by $g \in G(H)$ and $x \in P_{1, g}(H) . H_{n^{2}}$ is pointed with $G(H)=\left\{1, g, \ldots, g^{n-1}\right\}$. Now $1 \rightarrow g$ since $\Delta x=x \otimes 1+g \otimes x$; multiplying by $g^{k}$ as in 1.2 we also have $g^{k} \rightarrow g^{k+1}$. Thus $\Gamma_{C}$ is

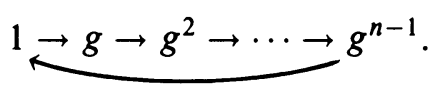

1.5 Example. Consider $C=U_{q}(s l(2))$, for $q \in k^{*}$ not a root of 1 , as described by Drinfeld and Jimbo; see [Dr]. That is, as an algebra

$$
H=k\left\langle E, F, K, K^{-1} \mid K E=q^{2} E K, K F=q^{-2} F K, E F-F E=\frac{K^{2}-K^{-2}}{q^{2}-q^{-2}}\right\rangle .
$$

Its coalgebra structure is determined by $K \in G(H)$ and $E, F \in P_{K^{-1}, K}(H)$. It is known that $H$ is pointed [M1, R] with $G(H)=\langle K\rangle$ and that the skew primitive elements are in the $k G(H)$-module spanned by $1, E$, and $F$ [M1, T1]. Moreover, for all $n, E K^{n}$ and $F K^{n} \in P_{K^{n-1}, K^{n+1}}(H)$. Consequently $\Gamma_{C}$ consists of two connected components:

$$
\begin{aligned}
& \cdots \rightarrow K^{-3} \rightarrow K^{-1} \rightarrow K \rightarrow K^{3} \rightarrow \cdots \\
& \cdots \rightarrow K^{-2} \rightarrow 1 \rightarrow K^{2} \rightarrow K^{4} \rightarrow \cdots .
\end{aligned}
$$

Thus $U_{q}(s l(2))$ is not link-indecomposable. 
We now make the connection to extensions of simple comodules. Let $M$ be any right $C$-comodule, with structure map $\Delta_{M}: M \rightarrow M \otimes C$, via $m \mapsto$ $\sum m_{(0)} \otimes m_{(1)} . M$ becomes a left $C^{*}$-module via $f \cdot m=\sum f\left(m_{(1)}\right) m_{(0)}$, for all $f \in C^{*}, m \in M$, and two comodules $M, N$ are isomorphic $\Leftrightarrow$ they are isomorphic as $C^{*}$-modules.

Now for each right comodule $M$ there is a unique minimal subcoalgebra $C(M)$ of $C$ such that $\Delta_{M}: M \rightarrow M \otimes C(M)$, that is, such that $M$ is a $C(M)$ comodule ([S2, p. 326] or [A, p. 129]). If $M$ is finite dimensional, so is $C(M)$, and if $M$ is a simple comodule, then $C(M)$ is a simple subcoalgebra of $C$ ([La, p. 354]: observe that by the minimality of $C(M), M$ is a faithful simple $C(M)^{*}$-module, and so $C(M)^{*}$ is a simple algebra). Moreover if $M \cong N$ are simple comodules, $C(M)=C(N)$. Conversely if $S$ is a simple subcoalgebra of $C$, then $S$ belongs to a simple right $C$-comodule $M$, unique up to isomorphism (namely, let $M$ be a simple left $S^{*}$-module). Summarizing, we have the following well-known result.

1.6 Lemma. There is a one-to-one correspondence between the set $\mathscr{M}$ of isomorphism classes of simple right $C$-comodules and the set $\mathscr{S}$ of simple subcoalgebras of $C$.

Now let $M, N$ be two simple right $C$-comodules. Then $\operatorname{Ext}^{C}(M, N) \neq 0 \Leftrightarrow$ there exists an exact sequence

$$
0 \rightarrow N \rightarrow P \rightarrow M \rightarrow 0
$$

where $P$ is an indecomposable right $C$-comodule. The Ext quiver of $\mathscr{M}$ is the directed graph which has as vertices the elements of $\mathscr{M}$ and has an arrow from $M$ to $N \Leftrightarrow \operatorname{Ext}^{C}(M, N) \neq 0$.

Recall that for a vector space $V$ with dual space $V^{*}$ and $U \subset V, U^{\perp}=$ $\left\{f \in V^{*} \mid f(U)=0\right\}$; similarly for $W \subset V^{*}$ we may define $W^{\perp} \subset V$.

1.7 Theorem. $\Gamma_{C}$ is isomorphic (as a directed graph) to the Ext quiver of simple (right) $\mathrm{C}$-comodules.

Proof. Lemma 1.6 gives a bijection between $\mathscr{S}$ and $\mathscr{M}$, and thus between the vertices of the graphs. To show the arrows correspond, we must show that for $M, N$ in $\mathscr{M}, \operatorname{Ext}^{C}(M, N) \neq 0 \Leftrightarrow C(M) \rightarrow C(N)$ as in 1.1. To do this, we first prove it is true when $C$ is finite dimensional.

Consider $M$ and $N$ as left $C^{*}$-modules as above and let $m=a n n M=$ $C(M)^{\perp}$ and $n=a n n N=C(M)^{\perp} ; m$ and $n$ are maximal ideals of $C^{*}$. By [S, 9.0.0], $\left(D^{\perp} E^{\perp}\right)^{\perp}=D \wedge E$, for any subspaces $D$ and $E$ of $C$. Thus

$$
C(N) \wedge C(M)=\left(C(N)^{\perp} C(M)^{\perp}\right)^{\perp}=(n m)^{\perp} .
$$

Also $C(M)+C(N)=m^{\perp}+n^{\perp}=(m \cap n)^{\perp}$. From 1.1, it follows that

$$
C(M) \rightarrow C(N) \Leftrightarrow n m \neq n \cap m .
$$

Now a standard argument for algebras shows that if $n$ and $m$ are maximal ideals of a finite-dimensional algebra $A$, and $M$ and $N$ are simple $A / m$ - and $A / n$-modules respectively, then $n m \neq n \cap m \Leftrightarrow \operatorname{Ext}_{A}(M, N) \neq 0$. Since $A=C^{*}$ is finite dimensional, every $A$-module is a $C$-comodule, and conversely. Thus $C(M) \rightarrow C(N) \Leftrightarrow \operatorname{Ext}_{C^{*}}(M, N) \neq 0 \Leftrightarrow \operatorname{Ext}^{C}(M, N) \neq 0$. This proves the theorem in the finite-dimensional case. 
We now show that in general, we may reduce to the finite-dimensional case. First assume that $\operatorname{Ext}^{C}(M, N) \neq 0$, and let $P$ be an indecomposable $C$ comodule with $0 \rightarrow N \rightarrow P \rightarrow M \rightarrow 0$. Since $M$ and $N$ are finite dimensional, so is $P$. Thus $C(N), C(P)$, and $C(M)$ all lie in some finite-dimensional subcoalgebra $D$ of $C$. Moreover, since $D$-subcomodules are also $C$-subcomodules, $N$ and $M$ are simple $D$-comodules and $P$ is an indecomposable $D$-comodule. Then $\operatorname{Ext}^{D}(M, N) \neq 0$, and so $C(M) \rightarrow C(N)$ in $\Gamma_{D}$ since $D$ is finite dimensional. By 1.1, clearly also $C(M) \rightarrow C(N)$ in $\Gamma_{C}$.

Conversely assume that $C(M) \rightarrow C(N)$ and choose $x \in C(N) \wedge C(M)$, $x \notin C(N)+C(M)$. Write $\Delta x \in \sum_{i} x_{i} \otimes C(M)+\sum_{j} C(N) \otimes y_{j}$ and let $D$ be the (finite-dimensional) subcoalgebra of $C$ generated by $x$, all $x_{i}$, all $y_{j}, C(N)$, and $C(M)$. Then $\Delta x \in D \otimes C(M)+C(N) \otimes D$ and so $C(M) \rightarrow C(N)$ in $D$. Since $D$ is finite dimensional, the above argument shows $\operatorname{Ext}^{D}(M, N) \neq 0$. However, any $D$-comodule is also a $C$-comodule, and thus $\operatorname{Ext}^{C}(M, N) \neq$ 0 .

We note that the finite-dimensional case of the above argument is essentially [CM, 1.1, Proposition]. Also the relationship $S+T=S \wedge T$ is mentioned in [XF], but only used for a criterion for determining when a coalgebra is a direct sum of irreducible ones.

At this point we compare $\Gamma_{C}$ with the equivalence relation of Shudo and Miyamoto [ShM]. For $S, T \in \mathscr{S}$, they define $S \sim T \Leftrightarrow$ either $S=T$, or when $S \neq T$, there exists a finite chain $S=S_{0}, S_{1}, \ldots, S_{n}=T$, with all $S_{i} \in \mathscr{S}$, such that $S_{i} \wedge S_{i+1} \neq S_{i+1} \wedge S_{i}$, all $i=0, \ldots, n-1$.

1.8 Lemma. For $S, T \in \mathscr{S}, S$ is connected to $T$ in $\Gamma_{C} \Leftrightarrow S \sim T$ in the sense of $[\mathrm{ShM}]$.

Proof. Using 1.1, it suffices to show that for $S \neq T,\{S \wedge T \neq S+T$ or $T \wedge S \neq S+T\} \Leftrightarrow S \wedge T \neq T \wedge S$. Equivalently,

$$
\{S \wedge T=S+T \text { and } T \wedge S=S+T\} \Leftrightarrow S \wedge T=T \wedge S .
$$

$(\Rightarrow)$ is clear. To show $(\Leftarrow)$, choose $c \in S \wedge T=T \wedge S$. We must show $c \in S+T$. By the definition of wedge,

$$
\begin{aligned}
\Delta c & \in(C \otimes S+T \otimes C) \cap(C \otimes T+S \otimes C) \\
& =(S+T) \otimes(S+T) .
\end{aligned}
$$

The equality is a standard vector-space argument using $S \cap T=(0)$. Since $c=(\mathrm{id} \otimes \varepsilon) \Delta c$, it follows that $c \in S+T$.

Thus the $[\mathrm{ShM}]$ equivalence classes correspond to the connected components of $\Gamma_{C}$. However, by looking only at equivalence classes of $\mathscr{S}$, a lot of information is lost: the directions of the arrows, as well as the possibility of an arrow from $S$ to itself, are not considered, nor is the connection to extensions of comodules.

\section{THE DECOMPOSITION THEOREM FOR COALGEBRAS}

In this section, we prove that any coalgebra can be decomposed as a direct sum of indecomposable components, each of which corresponds to a connected component of $\Gamma_{C}$. Although this result could be obtained by combining work 
of [K], [ShM], and Lemma 1.8, we give instead a fairly short, direct proof, which follows the outline of the proof for the cocommutative case given in [S1].

We also use the classical theorem of Brauer, which states that a finitedimensional algebra $A$ is indecomposable $\Leftrightarrow$ the Ext quiver $\Gamma_{A}$ of simple $A$-modules is connected (see for example, [P, p. 100]).

By a link-indecomposable component (L.I.C.), we mean a subcoalgebra $D$ which is maximal with respect to $\Gamma_{D}$ being connected.

2.1 Theorem. Let $C$ be any coalgebra. Then $C=\bigoplus_{\alpha} C_{\alpha}$, where the $C_{\alpha}$ are the link-indecomposable components of $C$.

Proof. We proceed by a series of steps as in the cocommutative case [S, 8.0.7].

(1) It is a well-known fact [S,8.0.3], [M,5.6.2] that if $C=\sum_{\alpha} C_{\alpha}$ is any sum of coalgebras $C_{\alpha}$, and $D$ is any simple subcoalgebra of $C$, then $D$ must lie in one of the $C_{\alpha}$.

(2) We claim that if $\left\{C_{\alpha}\right\}$ is a family of L.I. subcoalgebras of $C$ such that $\bigcap_{\alpha} C_{\alpha} \neq 0$, then also $\sum_{\alpha} C_{\alpha}$ is L.I.

For, $\bigcap_{\alpha} C_{\alpha}$ must contain a simple subcoalgebra, say $D$. Thus $D \subseteq C_{\alpha}$, for all $\alpha$. Let $E$ be any simple subcoalgebra of $\sum_{\alpha} C_{\alpha}$. By (1), $E \subseteq C_{\beta}$ for some $\beta$.

Thus both $D$ and $E$ are in $C_{\beta}$, which is L.I. Thus $D$ and $E$ are connected via a set of simples in $C_{\beta} \subseteq \sum_{\alpha} C_{\alpha}$. Similarly any simple $F$ in $\sum_{\alpha} C_{\alpha}$ is connected to $D$, and thus to $E$. Thus $\sum_{\alpha} C_{\alpha}$ is L.I.

(3) Any L.I. subcoalgebra is contained in a unique L.I. component. For, let $E$ be the sum of all L.I. subcoalgebras containing the given one; it is maximal (and unique) by construction, and is L.I. by (2).

(4) We claim that a sum of distinct L.I.C's is direct. For assume that $\left\{C_{\alpha}\right\}$ are distinct L.I.C's. If the sum is not direct, $C_{\beta} \cap\left(\sum_{\alpha \neq \beta} C_{\alpha}\right) \neq 0$ for some $\beta$. Let $D$ be a simple subcoalgebra in this intersection. Since $D \subseteq \sum_{\alpha \neq \beta} C_{\alpha}$, it follows by (1) that $D \subseteq C_{\gamma}$ for some $\gamma \neq \beta$. Thus $0 \neq D \subseteq C_{\beta} \cap C_{\gamma}$. Applying (2), $C_{\beta}+C_{\gamma}$ is L.I. By assumption each $C_{\alpha}$ is maximal L.I., and thus $C_{\beta}=C_{\beta}+C_{\gamma}$, a contradiction. This proves the claim.

(5) By (3) and (4), it remains only to show that the sum of the distinct L.I.C.'s is all of $C$. By local finiteness, any $c \in C$ lies in a finite-dimensional subcoalgebra; thus we may assume that $C$ is finite dimensional. Then $A=C^{*}$ is a finite-dimensional algebra, and so $A=\bigoplus_{i} A_{i}$ where the $A_{i}$ are indecomposable algebras. Thus $C \cong A^{*} \cong \bigoplus_{i} A_{i}^{*}$. We are now done by Brauer's theorem, since $\Gamma_{A_{i}} \cong \Gamma_{A_{i}^{*}}$ as noted in Theorem 1.7.

2.2 Corollary. $C$ is indecomposable $\Leftrightarrow \Gamma_{C}$ is connected.

Proof. $(\Rightarrow)$ If $\Gamma_{C}$ is not connected, then $C$ is not indecomposable by 2.1 .

$(\Leftrightarrow)$ Assume $\Gamma_{C}$ is connected, but that $C=D \oplus E$, for $D, E$ proper subcoalgebras of $C$. By assumption $\Gamma_{C}$ is connected; also by (1) in the proof of 2.1, every simple subcoalgebra of $C$ lies in $D$ or in $E$.

Thus we can find simple subcoalgebras $S \subseteq D$ and $T \subseteq E$ which are linked; say $S \rightarrow T$. Let $m=S^{\perp}$ and $n=T^{\perp}$ in $C^{*}$. Since $S \rightarrow T$, it follows by [S1, 9.0.0], as in 1.7, that $m n \neq m \cap n$, since $(m n)^{\perp}=S \wedge T \neq S+T=(m \cap n)^{\perp}$.

Now $C^{*} \cong D^{*} \oplus E^{*} \cong C^{*} / D^{\perp} \oplus C^{*} / E^{\perp}$. Under this isomorphism, since $m \supseteq$ $D^{\perp}$, we may find an ideal $I$ of $C^{*} / D^{\perp}$ such that $m=I \oplus C^{*} / E^{\perp}$. Similarly, 
since $n \supseteq E^{\perp}$, there is some ideal $J$ of $C^{*} / E^{\perp}$ such that $n=C^{*} / D^{\perp} \oplus J$. It follows that $m n=m \cap n$, a contradiction. Thus $C$ is indecomposable.

2.3 Remark. If $C$ is cocommutative and is link-indecomposable, then $C$ is irreducible. For, assume $C$ is not irreducible. Then $C$ contains two simple subcoalgebras with $S_{1} \rightarrow S_{2}$. If $x \in \Delta^{-1}\left(C \otimes S_{1}+S_{2} \otimes C\right), x \notin S_{1}+S_{2}$, consider the subcoalgebra $C_{1}$ generated by $x, S_{1}$, and $S_{2}$. Setting $m=S_{1}^{\perp}$ and $n=S_{2}^{\perp}$, we have (again as in 1.7) that $m n \neq m \cap n$. But $C_{1}^{*}$ is a finitedimensional commutative algebra, so is a finite direct sum of local rings. This is a contradiction. Thus we recover the classical result.

\section{Pointed Hopf algebras}

In this section we prove the crossed product decomposition mentioned in the introduction. We first need a known lemma.

3.1 Lemma. (1) If $C$ and $D$ are pointed coalgebras, then $C \otimes D$ is pointed and $G(C \otimes D)=G(C) \otimes G(D)$.

(2) If $f: C \rightarrow D$ is a surjection of coalgebras and $C$ is pointed, then $D$ is pointed and $G(D)=f(G(C))$.

Proof. (1) is [M2, 5.1.10] and (2) is [M2, 5.3.5].

In fact if $C$ and $D$ are pointed indecomposable, then $C \otimes D$ is also indecomposable. For, if $x \rightarrow y$ in $C$ and $z \in G(D)$, then $x \otimes z \rightarrow y \otimes z$ as in 1.2, using tensor products. Similarly, if $z \rightarrow w$ in $D$, then $y \otimes z \rightarrow y \otimes w$, and thus $x \otimes z \rightarrow y \otimes w$, for all $x, y \in G(C)$ and $z, w \in G(D)$. Thus $G(C \otimes D)$ is connected and so $C \otimes D$ is indecomposable by 2.2. It is false, however, that images of pointed indecomposable Hopf algebras are indecomposable: for, $k \mathbf{Z}_{2}=k 1+k g$ is an image of $H_{4}$, which is indecomposable by 1.4 and 2.2 .

We let $S$ denote the antipode of $H$. If $x \in G(H)$, then $S x=x^{-1}$ and thus $G(H)$ is actually a group.

3.2 Theorem. Let $H$ be a pointed Hopf algebra, and set $G=G(H)$. For each $x \in G$, let $H_{(x)}$ denote the indecomposable component containing $x$. Then:

(1) $H_{(x)} H_{(y)} \subseteq H_{(x y)}$ and $S H_{(x)} \subseteq H_{\left(x^{-1}\right)}$. In particular $H_{(1)}$ is a Hopf algebra.

(2) $G$ acts on $H_{(1)}$ by $x \cdot h=x h x^{-1}$, for all $x \in G, h \in H_{(1)}$.

(3) The set $N=G\left(H_{(1)}\right)$ is a normal subgroup of $G$.

(4) $H \cong H_{(1)}{ }_{\sigma} k(G / N)$, a crossed product of $H_{(1)}$ with the quotient group $G / N$, with cocycle $\sigma: G / N \times G / N \rightarrow N$.

Proof. (1) The argument generalizes those in [S1, §8.1] and [M2, 5.6.4]. If $x, y \in G$, then Lemma 3.1 implies that $H_{(x)} \otimes H_{(y)}$ is pointed indecomposable. Also multiplication $H_{(x)} \otimes H_{(y)} \rightarrow H_{(x)} H_{(y)}$ is a coalgebra surjection, and thus by $3.1 H_{(x)} H_{(y)}$ is pointed with $G\left(H_{(x)} H_{(y)}\right)=\left\{z w \mid z \in G\left(H_{(x)}\right), w \in G\left(H_{(y)}\right)\right\}$. Moreover a similar argument to the one after 3.1 shows that $G\left(H_{(x)} H_{(y)}\right)$ is connected. Thus $H_{(x)} H_{(y)}$ is link-indecomposable; since it contains $x y$, it must be contained in $H_{(x y)}$. It follows that $\left(H_{(1)}\right)^{2} \subseteq H_{(1)}$ and so $H_{(1)}$ is a bialgebra. It remains to show that $S H_{(x)} \subseteq H_{\left(x^{-1}\right)}$. Now $S$ is bijective since $H$ is pointed [M2, 5.2.11] and thus $S: H^{\text {cop }} \rightarrow H$ is a coalgebra isomorphism; here $H^{\text {cop }}$ is 
$H$ with the opposite coalgebra structure. Thus $H_{(x)}$ indecomposable implies that $S\left(H_{(x)}^{c o p}\right)$ is indecomposable. Since $x^{-1}=S x \in S\left(H_{(x)}^{c o p}\right)$, it follows that $S\left(H_{(x)}^{c o p}\right)$ is the indecomposable component containing $x^{-1}$. Thus $S H_{(x)}=$ $H_{\left(x^{-1}\right)}$.

(2) For each $x \in G$, the map $\phi_{x}: H \rightarrow H$ given by $h \mapsto x h$ is a coalgebra automorphism of $H$. Thus $\phi_{x}\left(H_{(1)}\right)=x H_{(1)}$ is the indecomposable component of $H$ containing $x$, and so $x H_{(1)}=H_{(x)}$. Similarly $H_{(x)}=H_{(1)} x$. Consequently $x H_{(1)} x^{-1}=H_{(1)}$.

(3) Now $N=\{x \in G(H) \mid x$ is connected to 1$\}$ since $H_{(1)}$ is the component containing $1 . N$ is a subgroup since $H_{(1)}$ is a Hopf algebra; however this is easy to see directly. For, $x \rightarrow y$ implies that $x z \rightarrow y z$ and $z x \rightarrow z y$ as in Remark 1.2. Thus if $x, y \in N$, so that $1 \sim x$ and $1 \sim y$, then $x y^{-1} \sim x y^{-1} y=x \sim 1$ and so $x y^{-1} \in N$. Thus $N$ is a subgroup. Similarly if $1 \sim x$ and $z \in G$, then $1 \sim z x z^{-1}$ and so $N$ is normal.

(4) The crossed product decomposition now follows from (2), (3), and Theorem 2.1. For, let $T$ be a set of distinct coset representatives of $N$ in $G$. Then $H=\bigoplus_{\bar{x} \in T} H_{(\bar{x})}=\bigoplus_{\bar{x} \in T} H_{(1)} \bar{x}$. Write $\bar{x} \bar{y}=\sigma(x, y) \overline{x y}$, where $\sigma(x, y) \in N$. Then if $h, k \in H_{(1)}$,

$$
(h \bar{x})(k \bar{y})=h \bar{x} k \bar{x}^{-1} \bar{x} \bar{y}=h(\bar{x} \cdot k) \sigma(x, y) \overline{x y} .
$$

Thus $H \cong H_{(1)} \#_{\sigma} k(G / N)$, a crossed product.

3.3 Example. We return to $U_{q}(s l(2))$, which we saw in 1.5 was not indecomposable. In this case the indecomposable component of 1 is

$$
H_{(1)}=k\left\langle E K, F K, K^{2}, K^{-2}\right\rangle
$$

with the same relations as before. Here $N=\left\langle K^{2}\right\rangle$ and so $G / N=\langle\bar{K}\rangle \cong \mathbf{Z}_{2}$, where $\bar{K}$ is the coset $K N$. The cocycle $\sigma: G / N \times G / N \rightarrow N$ is given by $\sigma(\bar{K}, \bar{K})=K^{2}$ and $\sigma(\bar{K}, \overline{1})=\sigma(\overline{1}, \bar{K})=\sigma(\overline{1}, \overline{1})=1$. Then

$$
U_{q}(\operatorname{sl}(2)) \cong H_{(1)} \#_{\sigma} k(G / N) \cong H_{(1)} \#_{\sigma} k \mathbf{Z}_{2} .
$$

We note that if we set $E_{1}=E K, F_{1}=K^{-1} F$, and $K_{1}=K^{2}$, then $H_{(1)}=$ $k\left\langle E_{1}, F_{1}, K_{1}, K_{1}^{-1}\right\rangle$ is the "new version" of $U_{q}(s l(2))$ which is now used in [L], [DK], [Tk]. Lusztig remarks in [L] that the new version is used " to avoid certain irrelevant fourth roots of 1 ". Theorem 3.2 suggests that $H_{(1)}$ is the more fundamental object since its group-like elements are connected.

A similar decomposition holds for $U_{q}(s l(n))$, as its skew primitives are also spanned over $k G(H)$ by the $E_{i}$ and $F_{i}$ (M. Takeuchi, private communication, following [Tk]).

3.4 Remark. The $H_{(1)}$ in $U_{q}(s l(2))$ above was also used in [MS] in determining the possible actions of $U_{q}(s l(2))$ on the polynomial ring $\mathbf{C}[x]$. In that paper, it seemed easier to first deal with $1, K^{2}$-derivations and then to consider the action of $K$, rather than to begin with $K^{-1}, K$ derivations. More generally, in considering actions of a pointed Hopf algebra $H$ on a ring $A$, Theorem 3.2 suggests that one could first consider the indecomposable case $H_{(1)}$, and then use known results about group crossed products to deal with the action of $G / N$ on $A$. 


\section{AdDED IN PROOF}

J. A. Green shows in Locally finite representations, J. Algebra 41 (1976), $137-171,(1.6 \mathrm{~b})$, that the indecomposable components of a coalgebra are the "blocks" with respect to an equivalence relation on the simple comodules using their injective covers. Although "adjacency" of the simple comodules may be different than in [ShM] (and in this paper), the equivalence classes are the same.

\section{REFERENCES}

[A] E. Abe, Hopf algebras, Cambridge Univ. Press, Cambridge, 1980.

[CM] W. Chin and I. Musson, Hopf algebra duality, injective modules, and quantum groups, Comm. Algebra 22 (1994), 4661-4692.

[DK] C. De Concini and V. G. Kac, Representations of quantum groups at roots of 1 , Progr. Math., vol. 92, Birkhäuser, Boston, 1990.

[Di] J. Dieudonné, Introduction to the theory of formal groups, Marcel Dekker, New York, 1973.

[Dr] V. G. Drinfeld, Quantum groups, Proc. Internat. Congr. Math., Berkeley, Vol. 1, 1986, pp. 789-820.

[GW] K. R. Goodearl and R. Warfield, An introduction to Noetherian rings, London Math. Soc. Stud. Texts, vol. 16, Cambridge Univ. Press, Cambridge, 1989.

[K] I. Kaplansky, Bialgebras, Lecture Notes in Math., University of Chicago, 1975.

[La] R. G. Larson, Characters of Hopf algebras, J. Algebra 17 (1971), 352-368.

[L] G. Lusztig, Modular representations and quantum groups, Contemp. Math., vol. 82, Amer. Math. Soc., Providence, RI, 1989, pp. 59-77.

[M1] S. Montgomery, Some remarks on filtrations of Hopf algebras, Comm. Algebra 21 (1993), 999-1007.

[M2] . _ Hopf algebras and their actions on rings, CBMS Lecture Notes, vol. 82, Amer. Math. Soc., Providence, RI, 1993.

[MS] S. Montgomery and S. P. Smith, Skew derivations and $U_{q}(\mathrm{sl}(2))$, Israel J. Math. 72 (1990), 158-166.

[P] R. S. Pierce, Associative algebras, Graduate Texts in Math., vol. 88, Springer-Verlag, New York, 1982.

[R] D. E. Radford, Irreducible representations of $U_{q}(g)$ rising from $\operatorname{Mod}_{C^{1 / 2}}^{\bullet}$, Quantum Deformations of Algebras and Their Representations, Israel Math. Conf. Proc., vol. 7, Amer. Math. Soc., Providence, RI, 1993.

[S1] M. E. Sweedler, Hopf algebras, Benjamin, New York, 1969.

[S2] _ Integrals for Hopf algebras, Ann. of Math. (2) 89 (1969), 323-335.

[ShM] T. Shudo and H. Miyamoto, On the decomposition of coalgebras, Hiroshima J. Math. 8 (1978), 499-504.

[Tf] E. J. Taft, The order of the antipode of a finite-dimensional Hopf algebra, Proc. Nat. Acad. Sci. U.S.A. 68 (1971), 2631-2633.

[Tk] M. Takeuchi, Some topics on $G L_{q}(n)$, J. Algebra 147 (1992), 379-410.

[XF] Y. H. Xu and Y. Fong, On the decomposition of coalgebras, Words, Languages, and Combinatorics (Kyoto 1990), World Scientific Publishing, River Edge, NJ, 1992, pp. 504-522.

Department of Mathematics, University of Southern California, Los Angeles, CaliFORNIA 90089-1113

E-mail address: smontgom@mtha.usc.edu 